Vol. 13 - Núm. 4- Abril 2003 MEDIFAM 2003; 13: 291-296

\title{
Calidad de vida profesional de los trabajadores de Atención Primaria del Área 10 de Madrid
}

R. SÁNCHEZ GONZÁlez, R. Álvarez Nido, S. LORENZO BordA ${ }^{1}$ Médicos de Familia. EAP San Blas. Parla. 'EAP Centro II. Getafe. Área 10. Madrid.

\section{RESUMEN}

Objetivo: conocer la calidad de vida profesional (CVP) percibida por los trabajadores de Atención Primaria de nuestra área de salud, identificando los principales componentes de la misma.

Diseño: estudio descriptivo transversal.

Material y métodos: ámbito: Atención Primaria (Área 10; Madrid). Participantes: todos los profesionales de Atención Primaria $(n=419)$. Mediciones: encuesta anónima autoadministrada que incluye el cuestionario CVP-35, además de variables demográficas y laborales.

Resultados: tasa de respuesta: $57,5 \%$. Edad media: $39 \pm 7$ años; mujeres: 172 (71,4\%); propietarios: 116 (48,1\%); turno de mañana: 121 (50,2\%); antigüedad media: $12 \pm 7$ años. La CVP media fue $5,2 \pm 1,8$. Los items con puntuación más baja fueron "posibilidad de promoción" $(2,34 \pm 1,57) y$ "conflictos con otras personas" $(3,38 \pm 2,16)$. Los items con puntuación más alta fueron "estoy capacitado para realizar mi trabajo" $(8,38 \pm 1,27) y$ "cantidad de trabajo" $(8,19 \pm 1,28)$. La percepción de la CVP es significativamente peor entre el personal médico que en el resto de profesionales $(p<0,05)$. No encontramos diferencias al analizar la CVP por el resto de variables.

Conclusiones: encontramos un nivel medio de CVP entre los trabajadores de nuestra área, aun-
Professional quality of life in sanitary personnel of Primary Health Care Area 10 in Madrid

\section{ABSTRACT}

Objective: to know the professional quality of life $(P Q L)$ perceived by the workers of Primary Health Care of our area of health, identifying the main components of the same one.

Design: cross-sectional descriptive study.

Material and methods: setting: Primary Health Care. Area 10. Madrid. Subjects: all the professionals of Primary Health Care $(n=419)$. Measurements: self administrated anonymous survey that includes the PQL-35 questionnaire, in addition to demographic and labour variables.

Results: answer rate: $57,5 \%$. Average age: 39 \pm 7 years; women: $172(71,4 \%)$; staff: 116 (48,1\%); morning turn: 121 (50,2\%); average antiquity: $12 \pm 7$ years. Mean PQL: 5,2 $\pm 1,8$. Item less valued was "possibility of promotion" (2,34 \pm $1,57)$ and "conflicts with other people" (3,38 \pm $2,16)$. Item more valued was "I am enabled to make my work" $(8,38 \pm 1,27)$ and "excess of work" $(8,19 \pm 1,28)$. The perception of the PQL is significantly smaller between the medical personnel who in the rest of professionals $(p<0,05)$. We did not find differences when analysing the PQL by the rest of variables.

Conclusions: we found a mean level of $P Q L$ between the workers of our area, although the valuation is worse between the doctors. One percei-

Los resultados preliminares de este trabajo se presentaron como comunicación oral en el XXI Congreso de la semFYC, San Sebastián, noviembre 2001.

Recepción: 22-08-02

Aceptación: 27-03-03 
que la valoración es peor entre los médicos. Se percibe excesiva carga de trabajo y pocos recursos aportados por la organización.

Palabras clave: Atención Primaria. Calidad de vida profesional. Personal sanitario. ves excessive service load and few resources contributed by the organisation.

Key words: Primary Health Care. Professional quality of life. Sanitary personnel.

\section{INTRODUCCIÓN}

La motivación laboral es el resultado de una serie de relaciones entre el esfuerzo individual, el rendimiento obtenido, las recompensas organizativas y los objetivos individuales ${ }^{1}$. Aunque cualquier trabajador es susceptible al desánimo y a la pérdida de entusiasmo por su trabajo, los que más riesgo tienen son los profesionales que mantienen un contacto directo con el público, especialmente en los ámbitos social, educativo y sanitario.

Hoy día, los profesionales sanitarios están sometidos a una gran sobrecarga asistencial por parte de unos pacientes cada vez más exigentes y críticos. Todo ello en medio de una creciente burocracia que apenas deja tiempo para desempeñar la labor asistencial y de la incomprensión de gestores sanitarios y políticos. Es decir, existen múltiples factores sociales, tecnológicos y económicos que obligan a que cada vez se exija mayor rendimiento a los profesionales sanitarios sin que, nunca, o casi nunca, se piense en su CVP. Esto es así a pesar de que, desde que los estudios de Maslach popularizaron el término burnout ${ }^{2,3}$, es conocida la relación entre la insatisfacción laboral en los profesionales asistenciales y la disminución de la calidad de las prestaciones ofrecidas por éstos, tanto cualitativa como cuantitativamente ${ }^{1,4,5}$. En otras palabras, los profesionales tienden a dar calidad al cliente en la misma medida en que perciben que son tratados por la organización en que trabajan ${ }^{4}$.

La CVP puede definirse como la experiencia de bienestar secundaria a la percepción de equilibrio entre las demandas de un trabajo profesional y los recursos disponibles para afrontarlas ${ }^{1}$. Aunque disponemos de diferentes instrumentos para cuantificarla, son escasos los estudios realizados en nuestro país en el ámbito de Atención Primaria, cuyo objetivo sea medir la CVP de sus trabajadores.

Por todo lo anterior, nos planteamos la realización de este estudio con el siguiente objetivo: conocer la CVP percibida por los trabajadores que conforman el núcleo básico de los equipos de Atención Primaria de nuestra área de salud, identificando los principales componentes de la misma.

\section{MATERIAL Y MÉTODOS}

Se trata de un estudio descriptivo transversal realizado en el ámbito de Atención Primaria en el Área 10 del INSALUD de Madrid, durante los meses de mayo y junio de 2001.

Se envió la encuesta a todos los profesionales que forman el núcleo básico de cada uno de los 18 equipos de Atención Primaria del área (médicos, enfermeras, auxiliares de clínica, administrativos y celadores). Se designó una persona responsable en cada equipo para recoger las encuestas contestadas y devolverlas por "correo interno" en el plazo de 1 mes.

Utilizamos el cuestionario de calidad de vida profesional de 35 ítems (CVP-35) validado para nuestro medio ${ }^{5}$. En él se evalúa la CVP mediante 35 preguntas cerradas que hacen referencia a la percepción que tiene el trabajador de las condiciones de su puesto de trabajo en aspectos como: incomodidades derivadas del trabajo (dimensión A, 5 ítems), soporte emocional por parte de los directivos (dimensión B, 10 ítems), carga de trabajo percibida (dimensión C, 5 ítems), recursos ligados al lugar de trabajo (dimensión D, 4 ítems), soporte social recibido (dimensión E, 3 ítems), motivación intrínseca (dimensión F, 4 ítems) y capacidad de realizar el trabajo (dimensión G, 3 ítems). Además, existe un ítem específico sobre calidad global en el trabajo. Cada pregunta se puede responder según una escala de 1 a 10 a la que se superpusieron como ayuda las siguientes categorías: nada (valores 1-2), algo (valores 3-4-5), bastante (valores 6-7-8) y mucho (valores 9-10). En cada encuesta incluimos además, variables demográficas (edad, sexo) y otras variables presumiblemente relacionadas con la calidad de vida profesional (centro de trabajo, categoría profesional, turno, situación laboral y antigüedad en el INSALUD).

Las respuestas se procesaron en una base de datos para su explotación mediante el paquete estadístico EPI-Info. Se describen medias y desviaciones estándar (DE) en variables cuantitativas y porcentajes en variables cualitativas. El valor medio de cada dimensión se ha obtenido a partir de la 
media de los ítems que la componen. La comparación de medias de una variable cuantitativa por otra cualitativa dicotómica se realizó con la t de Student. En los casos en que la variable cualitativa tenía más de 2 categorías, se utilizó el análisis de la varianza de 1 vía (ANOVA). Se consideró significativa la $\mathrm{p}<0,05$.

\section{RESULTADOS}

Obtuvimos un total de 241 encuestas (tasa de respuesta: 57,5\%). Obtuvimos respuesta de todos los equipos, excepto de uno, a pesar de que se insistió a través de comunicación personal (oral y escrita) con la persona de referencia. Dicho equipo no presenta diferencias en lo que respecta a distribución por turno y categoría profesional con el resto de los que componen nuestra área de salud, y fue considerado como no respuesta.

En la tabla I se muestran las características de la población respondedora según las variables estu-

\section{Tabla I}

CARACTERÍSTICAS DE LA POBLACIÓN RESPONDEDORA Y TASA DE RESPUESTA SEGÚN TURNO Y CATEGORÍA PROFESIONAL

\begin{tabular}{|c|c|c|c|}
\hline Variable & $\begin{array}{l}\text { Número } \\
\text { de casos }\end{array}$ & Porcentaje & $\begin{array}{l}\text { Tasa de } \\
\text { respuesta }\end{array}$ \\
\hline \multicolumn{4}{|l|}{ Edad } \\
\hline$<30$ años & 29 & 12 & \\
\hline 30-39 años & 93 & 38,6 & \\
\hline 40-49 años & 87 & 36,1 & \\
\hline 50-59 años & 15 & 6,2 & \\
\hline$>60$ años & 2 & 0,8 & \\
\hline $\mathrm{NC}$ & 15 & 6,2 & \\
\hline \multicolumn{4}{|l|}{ Sexo } \\
\hline Hombre & 63 & 26,1 & \\
\hline Mujer & 172 & 71,4 & \\
\hline $\mathrm{NC}$ & 6 & 2,4 & \\
\hline \multicolumn{4}{|l|}{ Categoría } \\
\hline Celador & 11 & 4,6 & 47,8 \\
\hline Administrativo & 44 & 18,3 & 58,6 \\
\hline Auxiliar clínica & 7 & 2,9 & 38,8 \\
\hline Enfermero & 79 & 32,8 & 54,1 \\
\hline Médico & 90 & 37,3 & 57,3 \\
\hline $\mathrm{NC}$ & 10 & 4,1 & \\
\hline \multicolumn{4}{|l|}{ Situación laboral } \\
\hline Fijo & 116 & 48,1 & \\
\hline Interino & 106 & 44 & \\
\hline $\mathrm{NC}$ & 19 & 7,8 & \\
\hline \multicolumn{4}{|l|}{ Turno } \\
\hline Mañana & 121 & 50,2 & 62 \\
\hline Tarde & 119 & 49,4 & 53,1 \\
\hline $\mathrm{NC}$ & 1 & 0,4 & \\
\hline
\end{tabular}

diadas, así como la tasa de respuesta según turno y categoría profesional, que han sido las únicas variables utilizadas como control, con el fin de garantizar el anonimato. La edad media fue de 39 años ( $\mathrm{DE}=7$ años; rango: $22-66$ años) y la media de antigüedad en el INSALUD, de 12 años (DE $=7$ años; rango: 1-34 años).

El resultado obtenido en cada uno de los ítems y las dimensiones analizadas se presenta en la tabla II. En conjunto, obtenemos una percepción media de la CVP $(5,28 \pm 1,87)$, que se ajusta al siguiente perfil: los trabajadores de Atención Primaria de nuestra área perciben un lugar de trabajo con bastantes recursos $(6,64 \pm 0,98)$, con incomodidad $(4,72 \pm 0,94)$, con elevada carga de trabajo $(7,01 \pm$ $0,79)$ y escaso apoyo por parte de los directivos $(4,31 \pm 1,04)$. Además, estos profesionales otorgan puntuaciones elevadas a su grado de motivación $(6,41 \pm 0,61)$, capacitación $(7,62 \pm 0,82)$ y apoyo social $(6,48 \pm 1,13)$.

Si analizamos los ítems de manera individual observamos que los tres que alcanzan una mayor puntuación son "estoy capacitado para realizar mi trabajo" $(8,38 \pm 1,27)$, "cantidad de trabajo" $(8,19$ $\pm 1,28)$ y "mi trabajo es importante para la vida de otras personas" ( $7,84 \pm 1,81)$, mientras que los tres menos valorados son "posibilidad de promoción" $(2,34 \pm 1,57)$, "conflictos con otras personas" $(3,38$ $\pm 2,16)$ y "mi empresa trata de mejorar la calidad de vida" $(3,4 \pm 2,12)$.

Al analizar las puntuaciones obtenidas por la pregunta específica sobre "calidad de vida en el trabajo" según la categoría profesional, observamos que el grupo con mejor percepción de su calidad de vida es el de auxiliares de clínica y el peor el de los médicos (Tabla III). Esta diferencia, estadísticamente significativa $(p<0,05)$, se mantiene si agrupamos a todos los profesionales no facultativos y los comparamos con los médicos. Sin embargo, no observamos diferencias significativas al analizar la "calidad de vida en el trabajo" en función del sexo, la edad, el turno de trabajo, la antigüedad laboral y la situación laboral (Tabla IV).

\section{DISCUSIÓN}

Hemos obtenido una tasa de respuesta menor al de otros trabajos que utilizaban similar estrategia de encuesta y encaminados a valorar tanto la $\mathrm{CVP}^{6,7}$ como la satisfacción laboral ${ }^{8-14}$ en trabajadores sanitarios. Sin embargo, nuestra tasa de respuesta es similar o superior a la obtenida en algunos estudios sobre estrés laboral y burnout ${ }^{15-17}$. Al tratarse de cuestionarios anónimos, desconocemos las causas de no respuesta, pero no podemos descartar que pudiera existir un sesgo de no respuesta ${ }^{18}$. Además, si se da el caso de que las personas menos satisfe- 
Tabla II

PUNTUACIONES MEDIAS DE LAS DIMENSIONES E ÍTEMS EVALUADOS EN EL CUESTIONARIO DE CALIDAD DE VIDA PROFESIONAL

\section{Dimensión evaluada}

\section{Media}

A) Disconfort derivado del trabajo

Interrupciones molestas

Consecuencias negativas para la salud

Falta de tiempo para la vida personal

Incomodidad física en el trabajo

Conflictos con otras personas

B) Soporte emocional de los directivos

Variedad en mi trabajo

Posibilidad de expresar lo que pienso y necesito

Apoyo de mis jefes

Posibilidad de que mis propuestas sean escuchadas y aplicadas

Satisfacción con el sueldo

Posibilidad de ser creativo

Mi empresa trata de mejorar mi calidad de vida

Reconocimiento de mi esfuerzo

Recibo información de los resultados de mi trabajo

Posibilidad de promoción

C) Carga de trabajo

Cantidad de trabajo

Estrés

Prisas y agobios

Presión recibida para realizar mi trabajo

Presión recibida para mantener la calidad del trabajo

D) Recursos ligados al lugar de trabajo

Mi trabajo es importante para la vida de otras personas

Carga de responsabilidad

Lo que tengo que hacer queda claro

Autonomía o libertad de decisión

E) Apoyo social

Apoyo de mi familia

Apoyo de mis compañeros

Apoyo de mis subordinados

F) Motivación intrínseca

Ganas de ser creativo

Orgullo del trabajo

Motivación

Satisfacción con el trabajo

G) Capacitación para realizar el trabajo

Estoy capacitado para realizar mi trabajo

Se exige capacitación para realizar mi trabajo

Desconecto al acabar la jornada

H) Calidad de vida global

Desviación estándar

$4,72 \quad 0,94$

$5,97 \quad 2,55$

$5,13 \quad 2,52$

$4,49 \quad 2,75$

$4,64 \quad 2,76$

$3,38 \quad 2,16$

$4,31 \quad 1,04$

$5,49 \quad 2,32$

$5,34 \quad 2,19$

$4,42 \quad 2,39$

$5,13 \quad 2,1$

$3,62 \quad 2,24$

$4,65 \quad 2,28$

$3,4 \quad 2,12$

$3,51 \quad 2,12$

$5,2 \quad 2,24$

$\begin{array}{ll}2,34 & 1,57\end{array}$

$7,01 \quad 0,79$

$8,19 \quad 1,28$

$6,28 \quad 2,53$

$\begin{array}{ll}7,41 & 2,17\end{array}$

$6,37 \quad 2,34$

$6,82 \quad 2,2$

$6,64 \quad 0,98$

$7,84 \quad 1,81$

$6,71 \quad 2,31$

$6,59 \quad 1,93$

$5,42 \quad 2,21$

$6,48 \quad 1,13$

$7,65 \quad 2,28$

$6,42 \quad 2,18$

$5,38 \quad 2,51$

$6,41 \quad 0,61$

$6,84 \quad 2,26$

$6,99 \quad 2,22$

$5,68 \quad 2,52$

$6,15 \quad 2,23$

$7,62 \quad 0,82$

$8,38 \quad 1,27$

$7,74 \quad 2$

$6,74 \quad 2,44$

$\begin{array}{ll}5,28 & 1,87\end{array}$

\begin{tabular}{|c|c|c|c|c|}
\hline \multicolumn{5}{|c|}{ CALIDAD DE VIDA PROFESIONAL SEGÚN CATEGORÍA PROFESIONAL } \\
\hline Categoría & Media & Desviación estándar & F de Snedecor & $\mathbf{p}$ \\
\hline Celadores & 5,1 & 1,52 & 2,66 & 0,03 \\
\hline Administrativos & 5,26 & 2,43 & & \\
\hline Auxiliares clínica & 6,57 & 1,13 & & \\
\hline DUEs & 5,66 & 1,42 & & \\
\hline \multirow[t]{2}{*}{ Médicos } & 4,88 & 1,99 & & \\
\hline & & & T de Student & $\mathbf{p}$ \\
\hline Médicos & 4,88 & 1,99 & 2,57 & 0,01 \\
\hline Resto & 5,53 & 1,76 & & \\
\hline
\end{tabular}


Tabla IV

\begin{tabular}{|c|c|c|c|c|}
\hline \multicolumn{5}{|c|}{ CALIDAD DE VIDA PROFESIONAL SEGÚN DIFERENTES VARIABLES } \\
\hline Categoría & Media & Desviación estándar & T de Student & $\mathbf{p}$ \\
\hline \multicolumn{5}{|l|}{ Sexo } \\
\hline Hombre & 5,37 & 1,73 & 0,61 & 0,54 \\
\hline Mujer & 5,2 & 1,93 & & \\
\hline \multicolumn{5}{|l|}{ Edad } \\
\hline$\geq 40$ años & 5,43 & 1,8 & 1,04 & 0,29 \\
\hline$<40$ años & 5,16 & 1,92 & & \\
\hline \multicolumn{5}{|l|}{ Turno } \\
\hline Mañana & 5,41 & 1,92 & 1,16 & 0,24 \\
\hline Tarde & 5,12 & 1,81 & & \\
\hline \multicolumn{5}{|l|}{ Antigüedad } \\
\hline$\geq 12$ años & 5,29 & 1,95 & 0,08 & 0,93 \\
\hline$<12$ años & 5,31 & 1,78 & & \\
\hline \multicolumn{5}{|l|}{ Situación } \\
\hline Fijo & 5,42 & 1,76 & 1,38 & 0,16 \\
\hline Interino & 5,07 & 1,96 & & \\
\hline
\end{tabular}

chas con su calidad de vida profesional no hubieran contestado la encuesta, deberíamos considerar que los resultados obtenidos fueran mejores que los reales.

Otra de las limitaciones de nuestro estudio se debe a que el cuestionario utilizado sólo es válido para medir percepciones, sin embargo no sirve para medir las expectativas de los trabajadores ${ }^{5}$. Aunque el conocimiento de éstas podría ser más importante a la hora de tomar decisiones, creemos que los resultados que hemos obtenido pueden ayudar a conocer la situación en la que nos encontramos y ser un buen punto de partida para la implantación de medidas encaminadas a apoyar a los profesionales para mejorar su CVP.

La calidad de vida profesional global tiene unos niveles aceptables, similar a la obtenida en un estudio realizado en Atención Primaria utilizando el mismo cuestionario ${ }^{6}$, aunque inferior a otro realizado en el ámbito hospitalario ${ }^{7}$. La comparación con otros trabajos que han medido la satisfacción laboral del personal sanitario en Atención Primaria se hace difícil ya que utilizan otros instrumentos de medida, que no hacen referencia expresa a la $\mathrm{CVP}^{8-14}$.

Si comparamos nuestros resultados con los obtenidos en un estudio similar llevado a cabo en Tarragona $^{6}$ observamos que nuestros profesionales refieren más disconfort en relación con el trabajo, menos apoyo por parte de la empresa, más carga de trabajo, menos recursos ligados al trabajo, menos apoyo social y menos motivación, con una capacitación superior.

Las bajas puntuaciones obtenidas por los ítems "reconocimiento de mi esfuerzo”, "mi empresa trata de mejorar mi calidad de vida", "apoyo de mis jefes" y "satisfacción con el sueldo" nos presenta a un grupo de profesionales que no perciben que la organización sanitaria se aproxime a sus necesidades profesionales de participación, creatividad y reconocimiento. Hacia la misma dirección apunta la gran diferencia existente entre las puntuaciones de "ganas de ser creativo" y "posibilidad de ser creativo". Todos los datos referentes a los aspectos laborales negativos, la falta de reconocimiento del trabajo personal y la escasa identificación con la empresa han sido señalados con anterioridad por otros autores ${ }^{6,7,12}$ y se relacionan con insatisfacción, agotamiento y desgaste en el trabajo. A esto hay que añadir que la cuestión sobre "posibilidad de promoción" es la que menos puntuación alcanza de todas las que componen la encuesta. Este dato coincide con lo señalado por otros estudios ${ }^{2,3,6,8,9,11-13}$ y es el reflejo de la percepción que los profesionales tienen de un sistema sanitario que favorece el estancamiento y en el que no se promueve la competencia profesional.

Por el contrario, los ítems con puntuación más alta son los que hacen referencia a la capacitación profesional y la carga de trabajo, como en otros estudios $^{6,8,9}$. Es necesario prestar atención a la elevada puntuación obtenida por la cuestión sobre carga de trabajo diaria, ya que es patente la relación entre presión asistencial excesiva y riesgo de desarrollar burnout ${ }^{10,14,15,17}$.

También confirmamos las diferencias existentes entre las distintas categorías profesionales, siendo los médicos los profesionales con peor percepción de su calidad de vida profesional y los auxiliares de 
clínica los profesionales con mejor percepción, como ya ha sido reflejado en otros trabajos ${ }^{67}$. Es llamativo que los profesionales que presentan una peor percepción de su calidad de vida sean aquéllos con mayor formación académica de todos los que componen un EAP. Esto puede ser debido a que, junto al mayor nivel de exigencia y responsabilidad al que están sometidos, existe un desequilibrio evidente entre la actividad para la que han sido formados y lo que ahora se les pide que hagan, primando los resultados "empresariales" de eficiencia, a costa de un esfuerzo que, la mayor parte de las veces, no es adecuadamente compensado.

Como conclusión, queremos señalar que los trabajadores de nuestra área perciben su lugar de trabajo con bastantes recursos, con alguna incomodidad, con bastante carga de trabajo y con escaso apoyo emocional por parte de sus directivos. A pesar de ello encontramos unos profesionales con bastante motivación, bastante capacitación y bastante apoyo social y que tienen una percepción media de su CVP.

Consideramos finalmente que el empleo de este tipo de herramientas por parte de las instituciones sanitarias debería ser una práctica tan habitual como lo es la utilización de encuestas de satisfacción del usuario, ya que nos permitirían conocer más sobre el estado de opinión de los trabajadores en relación con su empresa y con su calidad de vida laboral, con el objeto de introducir mecanismos correctores que prevengan el agotamiento profesional, una de los problemas de nuestro actual sistema sanitario público. Algunas de estas estrategias correctoras podrían estar ligadas al desarrollo de habilidades de afrontamiento en los profesionales y a la adecuación organizacional de las sobrecargas a las que están sometidos.

\section{CORRESPONDENCIA:}

Raúl Sánchez González

Avda Orovilla, 57, $6^{\circ} \mathrm{C}$

28041 Madrid

e-mail: Rayol@inicia.es

\section{Bibliografía}

1. García S, González JA. Factores de motivación de los profesionales de la salud en Atención Primaria. FMC 1995; $2: 1$.

2. Maslach C. Burnout: A social psychological analysis. En: Jones JW. The burnout syndrome. California: Berkely University of California; 1981.

3. Maslach C. Understanding burnout definitional inssues in analyzing a complex phenomenon. En: Paine WS, ed. Job, stress and burnout: Research, theory and intervention perspectives. Sage Publications, 1982.

4. Cabezas C. Síndrome de desgaste profesional, estrés laboral y calidad de vida profesional. FMC 1998; 5: 491-2

5. Cabezas C. La calidad de vida de los profesionales. FMC 2000; 7 (Supl. 7): 53-68.

6. Clúa JL, Aguilar C. La calidad de vida profesional y el orgullo de trabajar en la sanidad pública. Resultados de una encuesta. Aten Primaria 1998; 22: 308-13.

7. Galobart A, Grau J, Sicras A, García G. Satisfacción profesional. Med Clin (Barc) 1995; 105: 76.

8. Fernández MI, Villagrasa JR, Gamo M, Vázquez J, Cruz E, Aguirre MV, et al. Estudio de la satisfacción laboral y sus determinantes en los trabajadores sanitarios de un área de Madrid. Rev Esp Salud Pública 1995; 69: 487-97.

9. Fernández MI, Moinelo A, Villanueva A, Andrade C, Rivera M, Gómez JM, et al. Satisfacción laboral en los profesionales de Atención Primaria del área 10 del Insalud de Madrid. Rev Esp Salud Pública 2000; 74: 139-47.

10. Mira JJ, Vitaller J, Buil JA, Rodríguez J. Satisfacción y estrés laboral en médicos generalistas del sistema público de salud. Aten Primaria 1994; 14: 1135-40.

11. García S, Custey MA. Evaluación en la satisfacción de los profesionales de atención primaria. Medifam 1992; 2: 16-24.

12. Hidalgo I, Díaz RJ. Estudio de la influencia de los factores laborales y sociodemográficos en el desarrollo del síndrome de agotamiento profesional en el área de medicina especializada del Insalud de Ávila. Med Clin (Barc) 1994; 103: 408-12.

13. Cruz E, Vázquez J, Aguirre V, Fernández MI, Villagrasa JR, Andradas V. Evaluación de la satisfacción del personal de enfermería. Aten Primaria 1994; 13: 469-73.

14. Olivar C, González S, Martínez MM. Factores relacionados con la insatisfacción laboral y el desgaste profesional en los médicos de atención primaria de Asturias. Aten Primaria 1999; 24: 352-9.

15. De Pablo R, Suberviola JF. Prevalencia del síndrome de burnout o desgaste profesional en los médicos de atención primaria. Aten Primaria 1998; 22: 580-4.

16. Caballero MA, Bermejo F, Nieto R, Caballero F. Prevalencia y factores asociados al burnout en un área de salud. Aten Primaria 2001; 27: 313-7.

17. Prieto L, Robles E, Salazar LM, Daniel E. Burnout en médicos de atención primaria de la provincia de Cáceres. Aten Primaria 2002; 29: 294-302.

18. Sánchez JA, Saturno PJ. Las encuestas de satisfacción. En: Tratado de calidad asistencial en atención primaria. Tomo III. Dupont Pharma, 1998. p. 311-39. 